\title{
A SIX-YEAR SURVEY OF THE CASES SEEN AT A SCHOOL CARDIAC CLINIC
}

\author{
BY \\ JOHN APLEY and C. BRUCE PERRY \\ From the Departments of Child Health and Medicine, University of Bristol
}

(RECEIVED FOR PUBLICATION MARCH 15, 1954)

\begin{abstract}
Cardiac clinics, to which children may be referred by general practitioners or school medical officers, have now been established at many centres in this and other countries long enough for their value to be assessed.
\end{abstract}

It is generally agreed that for the child they are of value in several ways. First, a firm diagnosis of ' functional', 'physiological', or 'insignificant' murmurs can be made and the child spared needless restriction and invalidism. Second, cases of rheumatic heart disease can be kept under observation and guidance so that relapses may be recognized and treatment instituted as soon as possible (this applies particularly to relapses of carditis unassociated with arthritis or chorea) and the child advised regarding education and subsequent employment. Third, cases of congenital heart disease can be diagnosed as accurately as possible and the parents and school authorities advised as to any necessary restriction and about education and subsequent employment. Further, the possibility of corrective surgery can be assessed.

For general practitioners and school medical officers the clinics afford readily available advice and help in the management and care of the children.

For paediatricians or cardiologists in charge of such clinics a vast field is opened up for observational research on the natural history of congenital and rheumatic heart disease. These clinics also provide many fascinating diagnostic problems and are of great value in undergraduate and postgraduate teaching, since they bring together so many and such varied cardiac physical signs. Finally, the analysis of the cases referred to the clinic gives information as to the incidence of the various types of heart disease occurring in the community served. Some reports giving information of this nature about such clinics have appeared in North America (e.g., Robinson, Aggeler and Daniloff, 1948; Keith, 1951), but we have traced only one from the British Isles, that of Price (1949), who described a clinic in a semi-urban area during its first 18 months. It was therefore considered that a report from a clinic in an urban area might provide material of interest, the value of which would be enhanced by a follow-up survey of several years' duration.

The clinic in Bristol was started in 1927 by Dr. Carey Coombs to provide a service for the School Medical Department, and has been continued since. It would have been interesting to review the part the clinic has played over the 25 years of its existence, but there were insuperable difficulties. First, it must be admitted that the accuracy of diagnosis in both rheumatic and congenital heart disease has been increased in recent years, in part because of the cumulative experience from cardiac clinics. Thus, it has been shown clearly that many murmurs, even when occurring in children with a history of 'rheumatism', are in fact 'innocent' or 'functional' murmurs. Second, as a result of the intensive study of congenital heart disease over the past two decades, a fairly firm diagnosis can now be made, in the majority of cases, on a clinical basis. For these two general reasons comparisons over a period of 20 years or more are not valid. A third, and local, reason is that some of the Bristol records were lost as the result of enemy action in the war. For these various reasons, and to permit of a reasonable period of observation, only the cases referred to the clinic between 1943 and 1948 have been studied.

Essentially the clinic serves the Bristol City area, and patients living outside Bristol have been excluded from this study. The large majority of children who attended were referred from Bristol school clinics. The school population was 38,500 in 1943 rising to 48,700 in 1948.* In addition, some younger children were referred from pre-school clinics, and this group included a large proportion

* The school leaving age was raised from 14 to 15 years in 1948. For consistency, however, all children over 14 years of age have been excluded throughout the series. 
of the cases with congenital heart disease. A few children were referred for an opinion by the family doctor. The remainder attended for routine observation after being discharged from hospital where they had been in-patients.

Table 1 shows the number of new cases attending annually, which fluctuated round an average of 214 .

TABLE 1

\begin{tabular}{c|c} 
NEW CASES ATTENDING ANNUALLY \\
\hline Year & No. of New Cases \\
\hline 1943 & 237 \\
1944 & 221 \\
1945 & 223 \\
1946 & 176 \\
1947 & 194 \\
1948 & 235 \\
\hline Total & 1,286 \\
\hline
\end{tabular}

The majority of children attending fell into two age-groups, corresponding with the ages at which routine school medical examinations are carried out: $40 \%$ were between 10 and 13 years old, and $27.5 \%$ between 5 and 7 years. A further $15 \%$ were under 5 years of age.

\section{Methods of Assessment}

After taking the history from the accompanying parent or other relative, and carrying out a physical examination, ancillary investigations were made when they were considered necessary. At the first attendance, in about one-fifth of the cases, a radiograph of the chest was taken and this was followed by fluoroscopy in a small proportion. An electrocardiograph was taken in just over one-fifth of the total. The proportion in which these investigations were done was considerably increased at subsequent attendances. In any patient in whom active disease was suspected an erythrocyte sedimentation rate estimation was done. Other special examinations were obtained in a small number of patients. In some children, usually those with congenital heart disease, angiocardiography or/and cardiac catheterization was later performed.

Children with no abnormalities were discharged as a rule after being seen once, or at most twice; others continued under supervision for periods up to several years, usually until they reached the school-leaving age.

\section{Reason for Referral}

Suspected Heart Disease (551 Cases). In the largest single group of cases the child was referred because a cardiac abnormality had been suspected on routine examination.* In some a murmur had been timed and described, and in some cases a definite or tentative diagnosis of a functional murmur or organic (rheumatic or congenital) heart disease had been made. A few were referred with tachycardia or bradycardia or, more commonly, an arrhythmia (nearly always diagnosed at the clinic as simple sinus arrhythmia or, in a smaller number, as extrasystoles). More rarely the patient was referred for suspected cardiac enlargement, or with the vague label of 'cardiac weakness' or 'heart attacks'.

Among the 551 cases, rheumatic heart disease was diagnosed in 24 (six active and 18 quiescent) and congenital heart disease in 98. A definite diagnosis could not be made in 15 . In 243 a functional murmur was diagnosed. In the remaining 171 cases no abnormality was found in the heart.

History of Previous Rheumatic Fever (196 Cases). These patients have been divided into two groups, according to whether they had or had not been in hospital during the alleged attack of rheumatic fever. This separation appeared advisable because of the imprecise description of the illness in many instances where the child had been treated at home. It was suspected that in a considerable proportion of the latter there had not, in fact, been a genuine

* Fourteen patients were excluded from this group because there was also a history of rheumatic fever ( 8 cases) or chorea (6 cases).

TABLE 2

PATIENTS REFERRED WITH A HISTORY OF PREVIOUS RHEUMATIC FEVER

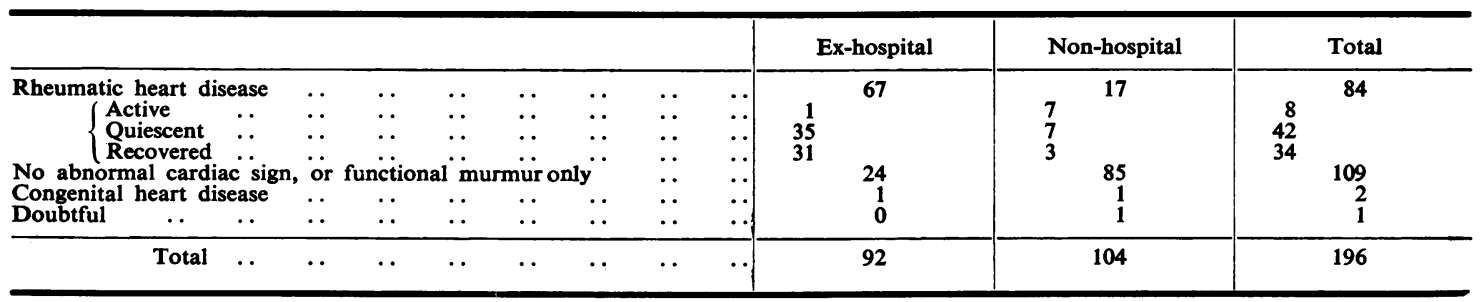


attack of acute rheumatism. This suspicion appeared to be confirmed by the considerably higher incidence of rheumatic heart disease in the hospital cases as compared with the others. As shown in Table 2, in the hospital group there was active or quiescent rheumatic heart disease in $39 \%$ compared with $13 \%$ in the non-hospital group. Another reason for the disparity between the two groups may be that doctors are more likely to refer a child with acute rheumatism to hospital if they find clinical evidence of cardiac involvement.

History of Previous Chorea (47 Cases). For the reason given in the previous group the patients in this group were also separated into hospital and non-hospital cases. It can be seen from Table 3 that in the former the incidence of active or quiescent rheumatic heart disease was $18 \%$, compared with $38 \%$ in the latter. This is very surprising, especially as there are probably more errors made in the diagnosis of chorea than of rheumatic fever. It is evident, whatever the reasons, that the factors operating as regards admission to hospital for rheumatic fever did not operate equally in cases of chorea.

Acute Rheumatism (55 Cases). Children suspected of having acute rheumatism are not, of course, usually referred as out-patients. Consequently, the number so referred included many admittedly doubtful cases.

In most of the patients in this group there was complaint of pains in the limbs or joints, sometimes with a history of swelling of the joints. Among the 55 patients acute rheumatism was confirmed in 14; nine of these had active carditis. One other child had quiescent rheumatic heart disease. In the majority of unconfirmed cases no positive diagnosis was made, though in some the symptoms were considered to be associated with a transitory upper respiratory infection. In two traumatic synovitis was diagnosed. One patient had a tuberculous knee and another pneumococcal arthritis.
Acute Chorea (105 Cases). Among the children referred because of suspected chorea, the diagnosis was confirmed in 19 (six with active carditis). The large majority of the remainder suffered from some form of nervous tic, but in a few no disorder was found.

'Chronic Rheumatism' (168 Cases). Various terms, such as 'chronic rheumatism', 'subacute rheumatism', 'muscular rheumatism' and 'growing pains', were applied more or less indiscriminately to children complaining of vague pains in the limbs. On close questioning the pains were found not to involve joints.

Among 168 patients in this group a cardiac abnormality was found in three. In one of these active rheumatic carditis was present; another had a patent ductus arteriosus and the third a congenital interventricular septal defect. One other patient was found to be suffering from Osgood-Schlatter disease. The remainder fell into the group of so-called 'growing pains' (Naish and Apley, 1951).

It is interesting to note that one child in this group, in whom no abnormality was found at the first examination, developed rheumatic fever with chorea and carditis a year later. In another, who had complained of limb pains for nearly three years, primary pulmonary tuberculosis was diagnosed seven months after the first examination.

Miscellaneous Complaints (164 Cases). As reasons for referring patients in this heterogeneous group the following may be quoted: vague pains with coryza or a febrile illness, pain in the abdomen or side, joint swellings, Still's disease, nodules, blue lips or fingers, giddiness, 'black-outs', dizzy spells, and underweight. In all, presumably, some cardiac or rheumatic condition had been suspected.

In the 164 patients forming this group there was one with congenital heart disease and there were four with rheumatic carditis (two active and two quiescent). In a few of the others some disease process was found, the least uncommon being a

TABLE 3

PATIENTS REFERRED WITH A PREVIOUS HISTORY OF CHOREA

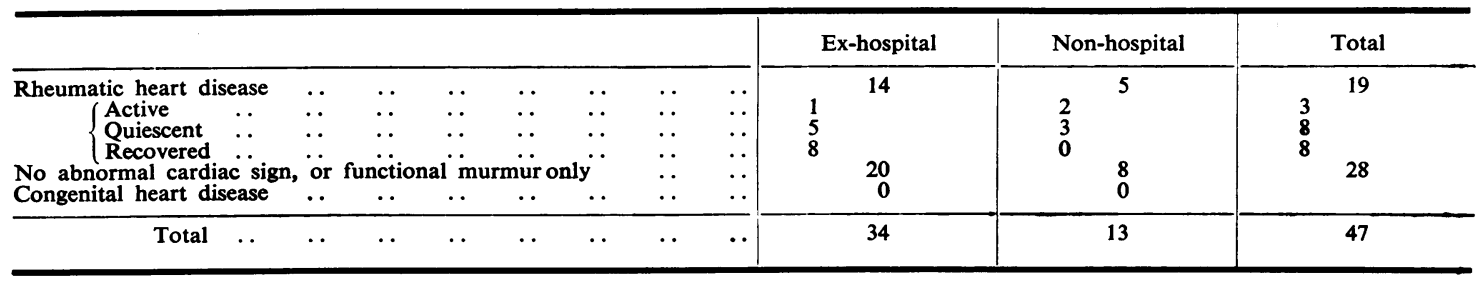


pulmonary disorder (such as asthma) or infection, or anaemia, with a few isolated examples of various other conditions.

\section{Rheumatic Heart Disease}

It was found convenient to classify cases of rheumatic heart disease into three groups: 'active', 'quiescent' and 'recovered'. The terms 'active' and 'quiescent' are self-evident; 'recovered' rheumatic heart disease was applied to those cases in which evidence (usually clinical and sometimes electrocardiographic) of cardiac involvement was found during the acute illness, but was no longer present when the patient attended the clinic. This diagnosis was usually possible only in children who had been hospital in-patients.

Among 148 children diagnosed as having rheumatic heart disease, in 35 there was active carditis, in 71 the rheumatic process was quiescent, and in 42 there appeared to be complete recovery.

Antecedents of Quiescent Disease. In the 71 patients comprising this group 42 had a history of rheumatic fever and eight of chorea. Among the 21 remaining, in four a history of scarlet fever was obtained, in four some apparently unrelated infection (such as pneumonia) had occurred, and in 13 there was no history of any disease which appeared to be relevant.

Age and Sex Incidence. Three children with rheumatic heart disease were seen at the age of 3 years, and two more at 4 years. A gradual rise in numbers then occurred in each age group until the age of 7; subsequently a fairly steady level was maintained, which fell again after the age of 13 .

There was no significant difference in sex incidence.

\section{Congenital Heart Disease}

One hundred and three children who attended were diagnosed as having congenital heart disease; the diagnoses are classified in Table 4.

TABLE 4

CASES WITH CONGENITAL HEART DISEASE

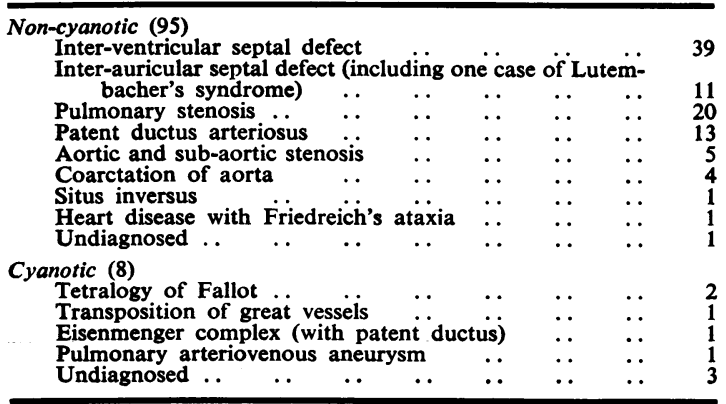

The diagnosis was almost invariably made on clinical and radiological (fluoroscopy) evidence, with electrocardiographic confirmation. In a small proportion of cases angiocardiography or/and cardiac catheterization was carried out subsequently. Most of the cases with patent ductus arteriosus, coarctation and tetralogy of Fallot were operated on. Postmortem examination was performed in five cases.

Age and Sex Incidence. Just under half the cases with congenital heart disease were referred under the age of 5 , i.e., before they had attended school. A further quarter of the total cases were referred in the two years after first going to school, as a result of school medical examinations. There was another small peak in the level of numbers referred at 10 years of age, coinciding with the second school medical examination.

The ratio of males to females was 62 to 41 .

\section{Functional Murmurs}

A diagnosis of a functional murmur was made in 303 children. The fact that 303 of the 1,286 children were found to have a functional murmur and that many of them were referred on account of this murmur illustrates one of the most important functions of a clinic of this sort. These murmurs frequently raise the suspicion of organic heart disease, and unless recognized as truly 'insignificant' may lead to much unnecessary anxiety and invalidism.

In this retrospective survey it was not found possible to assess accurately changes in the incidence of functional murmurs with age, one difficulty being that children with these murmurs did not invariably remain under continued observation. In some children who did continue to attend a functional murmur became audible at a variable time after the first clinic attendance, but in a rather larger number of patients who continued under observation functional murmurs were lost. On the whole, the incidence of these murmurs gradually fell with age.

\section{Undiagnosed Cases}

In 16 children no definite diagnosis was made, despite repeated observation. The problem in each instance was to decide whether organic heart disease was present or not. The cases fell almost equally into two groups, comprising possible rheumatic and possible congenital heart disease respectively. In most instances radiography, fluoroscopy and electrocardiography were carried out but were unhelpful. At the beginning of the six-year period angiocardiography and cardiac catheterization were not 
available as diagnostic aids; but even towards the end of the period they were not invoked, since the management of the case was not at issue in any instance.

\section{Major Changes in Diagnosis}

In a small proportion of children a preliminary or tentative diagnosis was confirmed or disproved during the course of subsequent examinations, because of modifications in the physical signs. These changes in diagnosis are not considered in detail, though they emphasize the value of continued observations.

In a small number of cases, however, a major change in diagnosis was made subsequent to the first examination, and these are summarized as follows.

In two children, with a history of previous rheumatic fever, new physical signs developed some months after the first examination (at which no abnormalities had been found in the heart) and the diagnosis of rheumatic heart disease was later made. In two other patients the diagnosis of congenital heart disease was subsequently changed to that of rheumatic heart disease. In three patients the diagnosis of rheumatic heart disease was changed to that of congenital heart disease. In nine children the diagnosis of congenital heart disease was changed to that of 'functional murmur', and in two the change was in the reverse direction. In most of these cases the change in diagnosis was made because of apparent alterations in the cardiac signs, but in a few a doubtful clinical diagnosis was influenced by subsequent fluoroscopic or electrocardiographic findings.

\section{Discussion}

Incidence. The true incidence of heart disease in children cannot be assessed from the attendances of patients referred to such a cardiac clinic. Though some estimates, based on clinical and ancillary appraisement (Robinson et al., 1948; Keith, 1951) or on necropsy figures (Keith, 1951; Apley, 1951) have been reported, there is a need for further reports from centres where access to unselected school and pre-school populations is completely comprehensive.

As regards the relative incidence of rheumatic and congenital disorders of the heart, there is some divergence of opinion. There has, however, been a striking decrease in recent years in the incidence of rheumatic heart disease, and also greater awareness and interest in the diagnosis of congenital heart disease. The ratio of rheumatic to congenital heart disease in school children as reported by Robinson et al. (1948) in San Francisco was 2.4 to 1.9. Keith (1951) in Toronto found a ratio of $44 \%$ rheumatic to $56 \%$ congenital in the 5-15 year agegroup. Both these computations are derived from 'heart registries' in which an attempt has been made to include all children in the district. In this country Price (1949) has published figures from a semi-urban cardiac clinic which, like the Bristol clinic, relies for its material on cases referred for an opinion. In his group the proportion of rheumatic to congenital cardiac disorders was $46 \%$ to $54 \%$. In the Bristol area the proportions were $59 \%$ to $41 \%$ if all cases of rheumatic heart disease (including 'recovered' cases) are included. If only active and quiescent cases are included the proportions of rheumatic and congenital cases are almost equal.

The Bristol figures for congenital heart disease are evidently an under-estimate, inasmuch as a number of children with cyanotic heart disease are kept from school, and hence from school clinics, or are referred elsewhere than to the clinic. The latter factor may also apply, to a much smaller extent, to non-cyanotic congenital heart disease. It is certain that the ratio of 95 acyanotic to 8 cyanotic is very misleading since it is well known that many patients with cyanotic congenital heart disease die in infancy. But even so, it is probable that more survive to school age and over than would appear from these figures.

Though the relative incidence of individual congenital anomalies as given by different authors varies widely, it is more or less generally agreed that the commonest single malformation is interventricular septal defect. Some of the discrepancies arise because children with certain anomalies may be referred more readily than others to clinics: a further discrepancy is evidently produced by variations in the criteria of diagnosis and in the use of ancillary methods of diagnosis. Thus, the high incidence of diagnosed pulmonary stenosis in the present series $(19 \%)$, and in that of Price in 1949 $(23 \%)$, should be contrasted with the very low incidence ( $1 \%$ ) reported by Keith (1951). The true incidence is clearly likely to be determined only in centres where there is access to unselected school and pre-school populations.

\section{Summary}

An analysis has been made of 1,286 children referred to a cardiac clinic for school children from 1943 to 1948. 
One hundred and forty-eight were diagnosed as having past or present rheumatic heart disease.

One hundred and three were diagnosed as having congenital heart disease ( 95 acyanotic).

Three hundred and three were diagnosed as having a 'functional' murmur.

\section{REFERENCES}

Apley, J. (1951). Bristol med.-chir. J., 68, 124.

Keith, J. D. (1951). In Modern trends in Paediatrics. ed. Parsons, L. pp. 75-122. London.
Naish, J. M. and Apley, J. (1951). Archives of Disease in Childhood,
26, 134. Price, R. Kemball (1949). Brit. med. J., 1, 515.

Robinson, S. J., Aggeler, D. M. and Daniloff, G. T. (1948). J. Pediat., 33, 49. 